African Crop Science Journal by African Crop Science Society is licensed under a Creative Commons Attribution 3.0 Uganda License. Based on a work at www.ajol.info/ and www.bioline.org.br/cs DOI: https://dx.doi.org/10.4314/acsj.v29i1.2

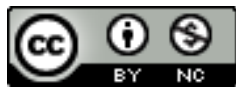

\title{
EFFETS DE LAFUMURE MINERALE ET DES PREDATEURS SUR LE NIVEAU D'INFESTATION DU COTONNIER PAR LE PUCERON (HOMOPTERA : APHIDIDAE) AU TOGO
}

\author{
P.K. AKANTETOU, P. TOZOOU ${ }^{2}$, M.E. BOKOBANA ${ }^{1}$, N.A. NADIO ${ }^{1}$, P. KILIMOU, K. KOBA ${ }^{1}$, \\ W. POUTOULI ${ }^{2}$ et K. SANDA ${ }^{1}$ \\ Institut Togolais de Recherche Agronomique (ITRA), BP 1163, Lomé, Togo \\ ${ }^{1}$ Laboratoire de Recherche sur les Agroressources et la Santé Environnementale (LARASE), Ecole \\ Supérieure d'Agronomie, Université de Lomé, BP. 20131, Lomé Togo \\ ${ }^{2}$ Laboratoire de Biologie Animale et de zoologie, Faculté des Sciences, Université de Lomé, B.P. \\ 1515 Lomé, Togo \\ Auteur correspondant: pakantetou@yahoo.fr
}

(Received 16 May 2020; accepted 25 January 2021)

\section{RESUME}

Dans la perspective de contribuer à l'élaboration d'une stratégie de gestion intégrée du puceron de cotonnier Aphis gossypii, les tests de l'effet de la fumure minérale et des prédateurs sur la variation des niveaux de population de ce dernier ont été étudiés. La collecte des données a été réalisée par un suivi à chaque trois jour sur plants de cotonnier (Gossypium hirsutum L.) installé en parcelles expérimentales à la Station d' Expérimentation Agronomique de l'Université de Lomé. Les résultats de la fluctuation de population d'Aphis gossypii ont révélé deux pics de pullulation durant le cycle du

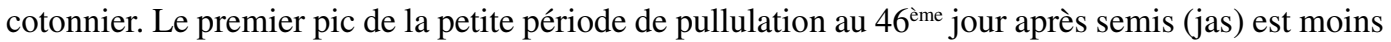
important numériquement. Le second pic de la grande période de pullulation au $86^{\text {ème }}$ jas est numériquement plus important et cause des dégâts considérables aux cotonniers. Les infestations et les niveaux de variation de populations au cours du cycle de la culture cotonnière sont très dépendants de la dose de fumure minérale appliquée La présence et le développement des prédateurs ont corrélé positivement avec le niveau d'infestation des plants par le puceron sans avoir un effet régulateur remarquable de la population de ce dernier.

Mots Clés: Aphis gossypii, infestation, prédateurs

\begin{abstract}
In order to contribute to the development of an integrated management strategy for cotton aphids (Aphis gossypii), tests of the effects of mineral manure and predators on the variation of its population levels were studied. Data collection was carried out in a three-day follow-up on cotton plants (Gossypium hirsutum L.) grown in experimental plots at the Station d'Expérimentation Agronomique de l'Université de Lomé in Togo. The results from population fluctuation of Aphis gossypii revealed two peaks of
\end{abstract}


swarming during the cotton plant cycle. The first peak of the small outbreak period on the 46th day after sowing (DAS) was less important numerically. The second peak of the great outbreak period on the 86th DAS was numerically larger and caused considerable damage to cotton plants. Infestations and levels of population variation during the cotton growing cycle were highly dependent on the dose of applied mineral fertilisers. The presence and development of predators positively correlated with the level of plant infestation by the aphids, without having a noticeable regulatory effect on their population.

Key Words: Aphis gossypii, infestation, predators

\section{INTRODUCTION}

La culture cotonnière occupe une place importante dans les activités économiques des agriculteurs au Togo, comme dans tous les pays de la sous-région ouest africaine producteurs du coton. Malgré la baisse de la production ces dernières années, le coton occupe le premier rang des produits agricoles de rente et le quatrième rang des produits d'exportation du Togo après le clinker, le ciment et le phosphate (MAEP, 2013). Cultivée en régime pluvial, cette culture cotonnière est une importante source de revenu monétaire pour les producteurs en milieu rural et de devises pour le pays. Ainsi, elle a un impact sur l'évolution des exploitations agricoles, sur les revenus monétaires et sur la réduction de la pauvreté (NSCT, 2013). Cependant, la pratique de la culture cotonnière rencontre beaucoup de contraintes. Le cotonnier est concurrencé par les mauvaises herbes et est très attaqué par les ravageurs et les maladies (Miranda et al., 2013). En Afrique subsaharienne, le faciès parasitaire du cotonnier a évolué au cours des deux derniers décennies d'une façon telle que les problèmes phytosanitaires pourraient remettre en cause la rentabilité et la durabilité de la culture, si des solutions adaptées à la situation ne sont pas proposées et appliquées rapidement (Deguine et Vaissayre , 2000). Au Togo, les pertes dues au complexe de ravageurs sans protection phytosanitaire peuvent varier entre 60 et $80 \%$ en moyenne (Akantetou, 2014). Parmi ces ravageurs, le piqueur suceur Aphis gossypii Glover (Homoptera : Aphididae) appelé puceron du cotonnier, devient très redoutable par rapport à sa densité et aux dégâts qu'il cause au cotonnier, aux autres plantes cultivées et ceci, à tous les stades de développement.

L'infestation du cotonnier par A. gossypii a lieu depuis la levée de la culture jusqu'à la récolte de coton-graine.

Ses dégâts sont de trois types : les dégâts trophiques qui sont directement liés aux piqûres des insectes ; les dégâts phytopathologiques causés par la transmission de maladies virales et les dégâts technologiques dus aux miellats qui souillent le coton graine en fin de campagne et provoquent le phénomène $\mathrm{du}$ « coton collant $»$ (Deguine et Ferron 2004). Ce dernier perturbe le marché international et inquiète tous les acteurs de la filière coton allant du producteur jusqu'au filateur (Frydrych et Hequet, 1997). Au Togo, le taux de coton collant est variable avec des niveaux élevés quelques années. Des taux de $11 \%$ en 1997 , $24 \%$ en 2001 et $31 \%$ en 2003 ont été enregistrés (ITMF, 2011). Cela explique la prise en compte du puceron A. gossypii dans les programmes de protection phytosanitaire de la culture cotonnière à base des produits chimiques de synthèse. Ces méthodes, insérées dans un programme calendaire ont quelquefois des limites. En effet, le niveau des infestations de la culture cotonnière par $A$. gossypii est élevé ces dernières années dans certaines zones agroécologiques du Togo (Akantetou et al., 2012). Malheureusement, les technologies innovantes, tel que le Cotonnier Génétiquement Modifié (CGM), mises au point et utilisées dans certains pays ne permettent pas le contrôle des ravageurs piqueurs suceurs 
(Bergé et Ricroch, 2010 ; Hofs et al., 2013) dont A. gossypii et le recours à l'utilisation des pesticides chimiques de synthèse reste la seule voie utilisée jusqu' aujourd' hui pour leur contrôle. A cet effet, il s'avère nécessaire de mettre au point une stratégie de lutte intégrée efficace et durable pour ce ravageur (Decoin et al., 1999). Celle - ci repose donc sur la connaissance de la dynamique de population, les facteurs qui l'affectent afin d'opter pour les méthodes de lutte sur seuils. En effet, la disponibilité de la sève en quantité et en qualité au niveau de la plante pourrait être un des facteurs favorables au niveau d'infestation de ce ravageur sur les cultures. Cette disponibilité de la sève serait liée aux doses des engrais minéraux appliquées aux cotonniers. Et pourtant un constat s'observe sur le terrain auprès des producteurs. Ces derniers, dans le but d'accroitre leurs productions et les rendements,choisissent d'augmenter les doses des engrais minéraux appliquées aux cotonniers. Ainsi, dans ce travail, nous avons déterminé les effets de l'augmentation des doses des engrais minéraux et des prédateurs sur le niveau d'infestation du cotonnier par le puceron Aphis gossypii en vue de contribuer à l'élaboration des méthodes de lutte intégrée contre ce ravageur.

\section{MATERILS ET METHODES}

L'étude a été conduite en 2012, sur une parcelle de cotonnier installée à la Station d'Expérimentation Agronomique de 1'Université de Lomé (SEAL ; N $6^{\circ} 22$ " ,22", E $1^{\circ} 13$ ", altitude $50 \mathrm{~m}$ ).

Le climat du site d'étude est de type tropical guinéen à 4 saisons. La pluviosité moyenne annuelle est de $1000 \mathrm{~mm}$ et la température moyenne annuelle est de $29^{\circ} \mathrm{C}$. La pluviosité au cours de l'année 2012 a été déficitaire par rapport à la moyenne ci-dessus. Le cumule annuel a été de $859 \mathrm{~mm}$ et la période de la conduite de l'essai (juillet - novembre) a été moins pluvieuse avec un cumule de 390 mm (Fig. 1). La moyenne des températures annuelles enregistrées a été de $29^{\circ} \mathrm{C}$.

Le dispositif adopté a été celui en blocs de Fisher à 4 objets et 4 répétitions. Les objets comparés ont porté sur différents doses d'engrais minéraux NPKSB (12-20-18-5-1) appliquées :

(i) le témoin sans fumure (SF),

(ii) la dose vulgarisée (D) : NPKSB (150 kg $\mathrm{ha}^{-1}$ )

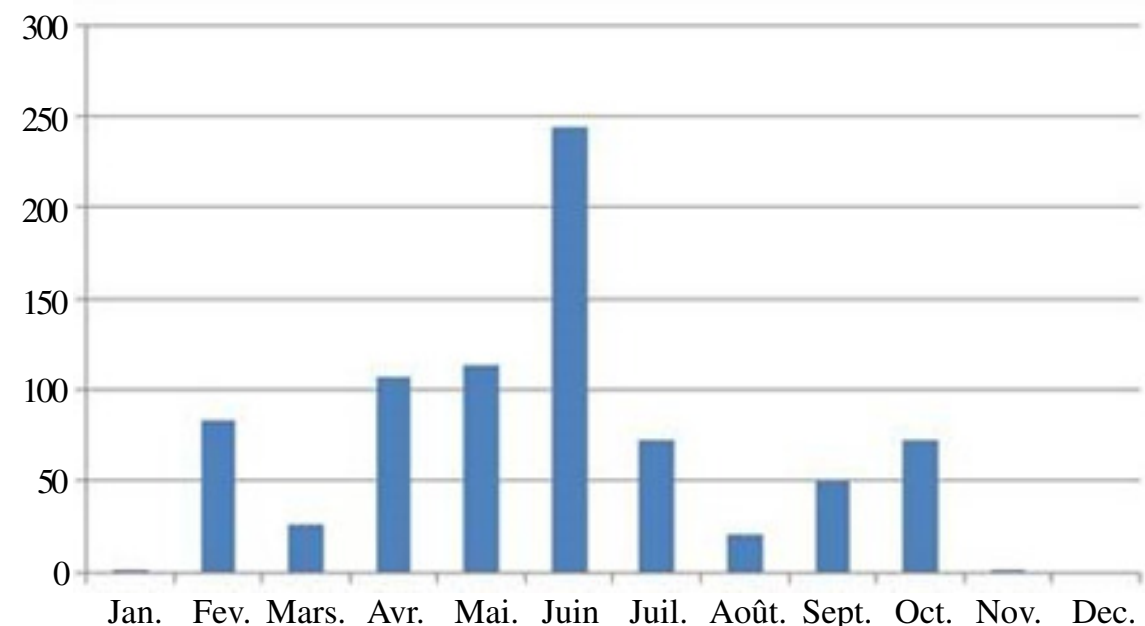

Figure 1. Cumul mensuel de hauteur de pluie en 2012 à la SEAL. 
(iii) la dose vulgarisée augmentée de sa moitié $(\mathrm{D}+\mathrm{D} / 2): \mathrm{NPKSB}\left(225 \mathrm{~kg} \mathrm{ha}^{-1}\right)$

(iv) le double de la dose vulgarisée (Dx2) : NPKSB (300 kg ha-1)

La parcelle élémentaire a une superficie de 14, $4 \mathrm{~m}^{2}$. L'infestation des plants par le puceron et ses prédateurs a été naturelle.

La variété de cotonnier installé a été la STAM 129A, créée et actuellement vulgarisée au Togo. Le semis a été effectué sur billon, dans un schéma de $0,8 \mathrm{~m}$ x 0,3 $\mathrm{m}$. Un sarclage et un buttage ont été réalisés respectivement

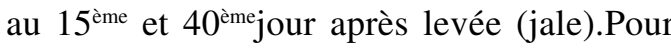
chaque objet, le NPKSB a été appliqué au $20^{\text {ème }}$ jale et une dose de $50 \mathrm{kgha}^{-1}$ d'Urée (46\% de $\mathrm{N}$ ) appliquée au $40^{\text {ème }}$ jale.

Les observations et la collecte des données ont porté sur le comptage des insectes, le puceron et ses principaux auxiliaires (coccinelles, syrphes et chrysopes) tous les trois jours, sur 20 plants choisis au hasard sur chaque parcelle élémentaire. Le cycle du cotonnier a couvert la période du 26 juin au 26 novembre 2012. Les observations pour la collecte des données ont été réalisées sur la période du 26 juillet au 26 novembre 2012. Le comptage des pucerons aptères et des prédateurs a été fait sur cinq feuilles terminales du plant choisi. Une loupe oculaire portative de $4,9 \mathrm{~cm}^{2}$ a été utilisée pour le comptage des larves.

\section{Traitement des données}

Taux d'évolution de la population d'A. gossypii sur cotonniers. Le calcul des taux d'évolution $(\mathrm{t})$ a permis de suivre la fluctuation des niveaux de la population de pucerons sur les cotonniers suivant les doses de fumure appliquées et dans le temps. Le taux est négatif si la valeur d'arrivée est inférieure à la valeur de départ et positif dans le cas contraire. Il peut aussi dépasser +1 si la valeur d'arrivée est supérieure au double de la valeur de départ. Le taux d'évolution s'exprime par la formule suivante
$t=\frac{V A-V D}{V D}$

$\mathrm{VD}=$ nombre de pucerons observés le jour $\mathrm{j}_{1}$

$\mathrm{VA}=$ nombre de pucerons observés le jour $\mathrm{j}_{1+\mathrm{n}}$

n = Nombre de jours après l'observation de départ

Le taux d'évolution permet aussi de calculer le coefficient multiplicateur (c) entre les deux valeurs considérées suivant la formule :

$\mathrm{C}=1+\mathrm{t}$

Analyses statistiques. Les analyses statistiques des moyennes du nombre de pucerons observé ont été réalisées à l'aide du logiciel XLSTAT 7.5.2. Le test de NewmanKeuls a permis de discriminer les groupes homogènes de moyennes des différentes données dans l'ordre décroissant. La signification est considérée au seuil de $5 \%$. A l'aide du tableur Excel 2007, les données de fluctuation de populations exprimées en nombre de pucerons et prédateurs sur 100 feuilles de plants de cotonnier sont représentées sous forme graphique (courbes, histogrammes) décrivant la tendance de l'évolution temporelle des infestations.

L'analyse de variance, le test $F$ et le modèle de régression linéaire ont permis de déterminer la corrélation $\mathrm{R}$ et un coefficient de détermination $R^{2}$ entre l'évolution du nombre de puceron observé dans le temps et les différentes doses de fumure minérale appliquées.

\section{RESULTATS}

Fluctuation de la population d' $A$. gossypii suivant les doses de fumure minérale. Les niveaux d'infestations des cotonniers par $A$. gossypii (Fig. 2) ont connu une variation suivant les quantités de fumure minérale appliquées. 


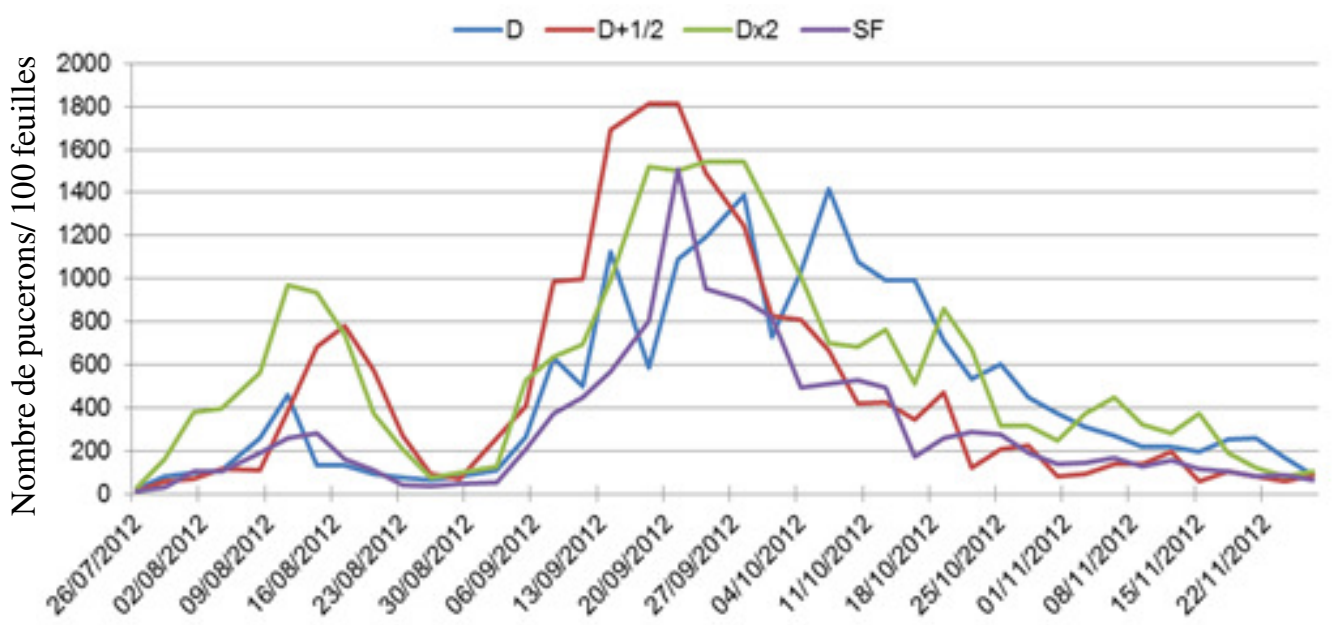

Dates des observations

Figure 2. Evolution comparée des niveaux d'infestation du cotonnier dans le temps suivant les doses de fumure minérale.

Dans tous les cas, les infestations ont connu une variation temporelle caractérisée par deux pics assez remarquables. La population d'A. gossypii a connu deux périodes de pullulation, observées à partir du $46^{\text {ème }}$ et $86^{\text {ème jour après }}$ semis (jas), durant le cycle du cotonnier. La première est intervenue pendant la phase de croissance de la culture et a été caractérisée par des pics relativement moins importants que ceux de la seconde. Ces pics ont évolué dans l'ordre croissant des quantités de fumures minérales apportées aux plants.

Les cotonniers qui n'ont pas reçu un apport de fumure minéral (SF) ont connu une augmentation de populations avec un pic qui a

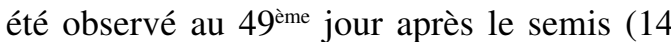
août) où plus de 280 pucerons ont été dénombrés sur un échantillon de 100 feuilles subterminales (Fig 2 courbe SF). Le taux d'évolution ( $\mathrm{t}$ ) a été de +22 pour un coéfficient multiplicateur (c) de 23 (Tableau 1). Une baisse des populations est observée jusqu'à un niveau minimum de près de 36 pucerons sur 100

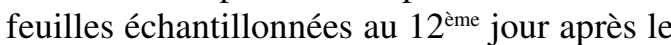
pic pour $\mathrm{t}=-0,87$ et $\mathrm{c}=0,13$. Une nouvelle croissance de la population a repris d'une manière exponentionnelle pour un deuxième pic avec 1509 pucerons observés au 86 ${ }^{\text {ème jour }}$ après semis (21 septembre) pour $\mathrm{t}=+40,92$ et $c=41,92$. Après ce deuxième pic, le niveau de populations a connu une regression jusqu'à la dernière observation réalisée au $154^{\text {ème jours }}$ après semis (jas) où le niveau de populations a été de 64 pucerons par 100 feuilles pour $\mathrm{t}=$ 0,96 et $\mathrm{c}=0,04$ (Tableau 1).

Le traitement qui a reçu la fumure minérale suivant la dose recommandée aux producteurs de coton (D) a connu une évolution plus rapide et un niveau de populations élevé. Ainsi, le premier pic a été observé au $46^{\mathrm{me}}$ jas (11 août) avec 460 pucerons observés par 100 feuilles (Fig. 2 courbe D) pour un taux d'évolution de +20,9 et un coefficient multiplicateur de 21,9 (Tableau 1). Après ce pic, une diminution de la pullulation a été observée et le niveau de populations le plus bas a été de 66 pucerons avec $\mathrm{t}=-0,86$ et $\mathrm{c}=0,14$. Cette baisse a été suivi d'un nouvel acroissement de la population mais en dents de scies donnant trois pics progressifs rapprochés très remarquables (Fig 2 courbe D). Sans considérer dans les détails les différentes variations au cours de cette phase, le pic le plus élevé a été observé au $102^{\text {ème }}$ jas (7 octobre) avec 1416 pucerons pour $\mathrm{t}=+20,45$ et $\mathrm{c}=21,45$. Le niveau de populations, après ce pic, a baissé jusqu'à la 
TABLEAU 1. Taux d'évolution des niveaux de population suivant la dose de fumure minérale.

\begin{tabular}{lccc}
\hline Niveaux de traitement & $\begin{array}{c}\text { Durée d'évolution } \\
\text { (jour)* }\end{array}$ & $\begin{array}{c}\text { Taux d'évolution } \\
\text { de la population ** }\end{array}$ & $\begin{array}{c}\text { Coefficient } \\
\text { multiplicateur *** }\end{array}$ \\
\hline SF & 19 & +22 & 23 \\
& 12 & $-0,87$ & 0,13 \\
& 25 & $+40,92$ & 41,92 \\
& 67 & $-0,96$ & 0,04 \\
D & 16 & $+20,9$ & 21,9 \\
& 15 & $-0,86$ & 0,14 \\
& 41 & $+20,45$ & 21,45 \\
& 51 & $-0,94$ & 0,06 \\
D+D/2 & 21 & $+77,1$ & 78,1 \\
& 12 & $-0,91$ & 0,09 \\
& 21 & $+24,18$ & 25,18 \\
& 67 & $-0,95$ & 0,05 \\
Dx2 & 16 & $+31,23$ & 32,23 \\
& 15 & $-0,92$ & 0,08 \\
& 28 & $+19,89$ & 20,89 \\
& 64 & $-0,93$ & 0,07 \\
\hline
\end{tabular}

* Temps mis pour passer d'un niveau bas de population à un pic et vis versa

** Quantification de la grandeur numérique de la population entre deux dates

*** Permet de calculer la valeur numérique de la population connaissant celle du départ ou celle d'arrivée

dernière observation réalisée au $154^{\text {ème }}$ jas avec une population de 89 pucerons sur 100 feuilles avec $\mathrm{t}=-0,94$ et $\mathrm{c}=0,06$.

Le traitement avec la dose recommandée plus la moitié $(\mathrm{D}+\mathrm{D} / 2)$ a connu le premier pic

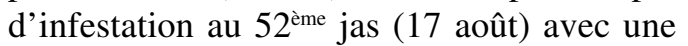
population de 781 pucerons (Fig. 2 courbe D $+\mathrm{D} / 2$ ) pour $\mathrm{t}=+77,1$ et $\mathrm{c}=78,1$ (Tableau 1). Le niveau de population a connu ensuite une diminution en douze jours jusqu'à un niveau minimal de 72 pucerons pour $\mathrm{t}=-0,91$ et $c=0,09$. La nouvelle croissance de la population a repris d'une manière rapide et a atteind un deuxième pic le plus grand au $86^{\text {ème }}$ jas (21 septembre) avec 1813 pucerons $(\mathrm{t}=$ $+24,18$ et $\mathrm{c}=25,18)$. La baisse de la population qui est intervenue après ce grand pic a été progressive avec 85 pucerons observés sur 100 feuilles au $154^{\text {ème }}$ jas avec $\mathrm{t}=-0,95$ et $\mathrm{c}$ $=0,05$.
Les plants de cotonniers qui ont reçu la double dose de la dose de fumure minérale vulgarisée (Dx2) ont connu un premier pic d'infestation au 46 ${ }^{\text {ème }}$.jas (11 août) avec une population maximale de 967 pucerons (Fig. 2 courbe Dx2 ) avec un $\mathrm{t}=+31,23$ et $\mathrm{c}=32,23$ (Tableau 1). Ce pic a été suivi d'une baisse jusqu'à un niveau minimum d'infestaion avec 74 pucerons observés au $15^{\text {ème }}$ jours après ce premier pic. La reprise de l'acroissement a été progressive pour atteindre un second pic en plateau sur une période de près d'un mois $(\mathrm{t}=$ $+19,89$ et $\mathrm{c}=20,89$ ) avec 1546 pucerons. La baisse du nombre de pucerons a été observée ensuite jusqu'au $154^{\mathrm{ème}}$ jas où la dernière observation a donné plus de 101 pucerons sur 100 feuilles observées avec un taux d'évolution de - 0,93 pour un coéfficient multiplicateur de 0,07 (Tableau 1). 
Effets fumure minérale et des prédateurs sur le niveau d'infestation

Comparaison des nombres moyens ont montré que de la levée au 30ème jas, le mensuels de pucerons par 100 feuilles de nombre de pucerons par 100 feuilles est très cotonniers suivant la dose croissante de faible. Des pucerons ont été observés sur fumure minérale. Les Variations moyennes quelques feuilles seulement de la levée jusqu'au mensuelles du nombre de pucerons suivant les début de la pleine croissance des plants. Les différentes doses de fumure minérale (Fig. 3) résultats d'analyse statistique de la variance
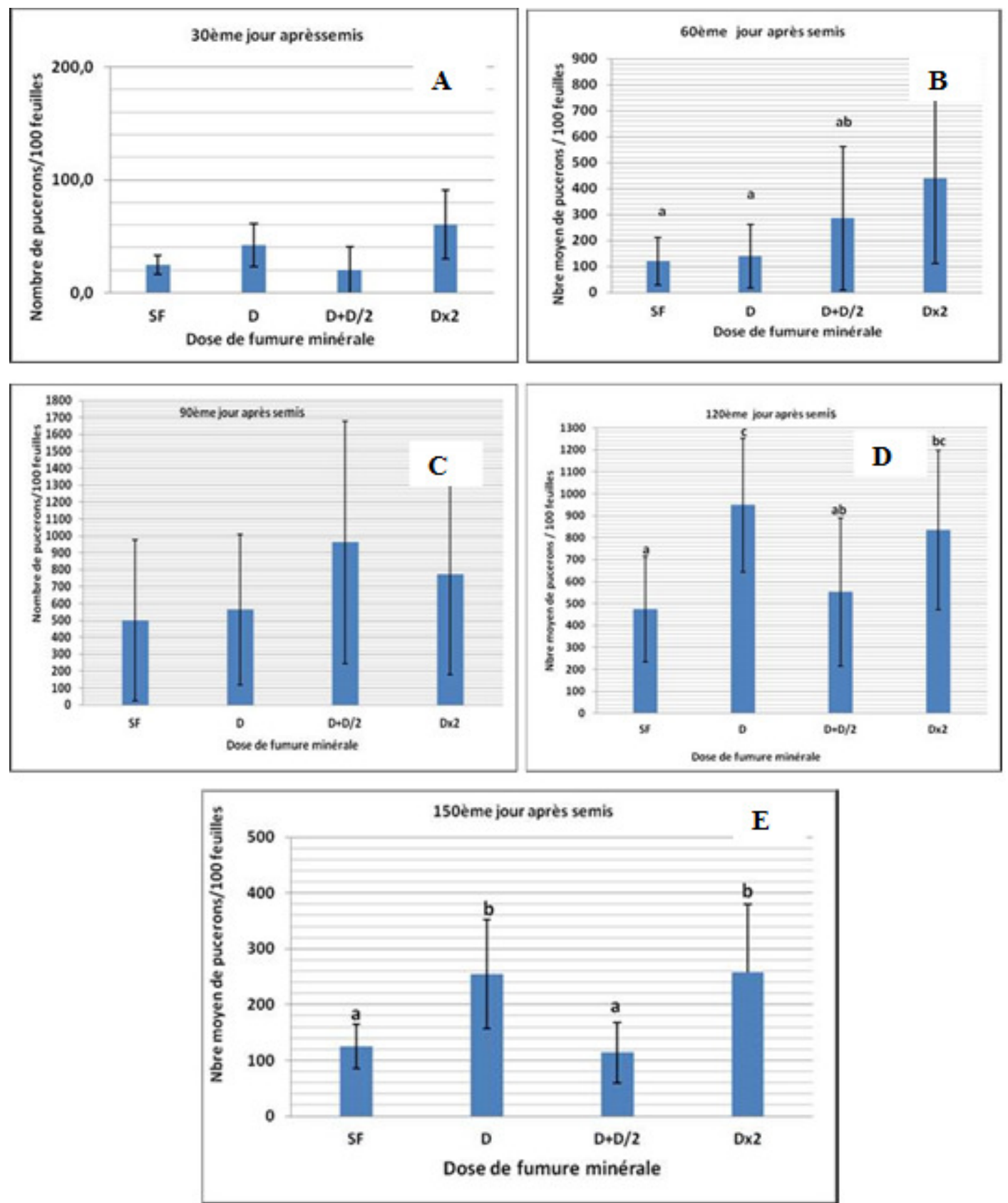

Figure 3. Variations moyennes mensuelles du nombre de pucerons suivant les différentes doses de fumure minérale à 30ème jas (A), 60ème jas (B), 90ème jas (C), 120ème jas (D), et 150ème jas (E). 
ont montré qu'il n'y a pas eu de différence significative entre les niveaux d'infestation des cotonniers des différents traitements de la levée au 30ème jas $(\mathrm{F}=3,02 ; \mathrm{dl}=3 ; 12, \mathrm{P}=0,07$; Fig. 3 A). Par contre, les résultats d'analyse de variance des données obtenues du 30 au 60 ème jas ont donné des différences très significatives entre les niveaux de populations observés sur les plants de cotonniers par rapport aux différentes doses de fumure minérale ( $\mathrm{P}=0,007$; Fig. $3 \mathrm{~B})$. Le test de Newman Keuls a révélé que la double dose de fumure minérale avec un niveau d'infestation moyen de 440 pucerons par 100 feuilles est nettement supérieure aux niveaux d'infestations observés sur les plants des autres traitements. Les plants des traitements SF et D ont subi un niveau d'infestation statiquement équivalent. Le résultat observé montre que le niveau de populations de pucerons a augmenté avec l'augmentation de la dose de fumure minérale (Fig. 3 B). Au stade floraison et fructification 60 à 90ème jas, les niveaux d'infestations ont été élevés dans les différents traitements. L'analyse de variance n'a pas révélé de différences significatives entre les niveaux de populations observés sur les plants de cotonniers soumis aux différents traitements $(P=0,21$; Fig. $3 \mathrm{C})$. En pleine fructification et au début d'ouverture des capsules (90 à 120ème jas), On a observé des différences très significatives $(\mathrm{P}=0,005)$ et la discrimination des moyennes avec le test de Newman Keuls a montré que les plants du traitement SF ont connu un niveau d'infestation nettement inférieur à ceux des traitements ayant reçu la fumure minérale (Fig. 3 D). Dans la phase de pleine ouverture des capsules (120 à 150ème jas), les niveaux d'infestation des plants de cotonnier suivant les différents traitements ont été significativement différents $(\mathrm{P}<0,0001)$ avec deux classes homogènes. Les plants des traitements $\mathrm{SF}$ et $\mathrm{D}+\mathrm{D} / 2$ ont été moins infestés par rapport aux plants des traitements D et Dx2 (Fig. 3 E)
Variation moyenne de populations de pucerons dans le temps suivant le modèle de régression linéaire. L'analyse de variance et le test $\mathrm{F}$ ont montré que le modèle de régression linéaire est significatif et de meilleure qualité avec une très forte corrélation $\mathrm{R}$ et un coefficient de détermination $R^{2}$ très bon. Ce dernier a révélé que la variation du niveau d'infestation des plants de cotonniers à toutes les phases d'accroissement de la population et pour tous les différents niveaux de traitements est très significativement corrélée au facteur temps (évolution de l'infestation du puceron sur les cotonniers au fil du temps). Par contre, pour les phases de régression des niveaux de populations, le modèle a donné des constantes significatives révélant ainsi qu'il existe d'autres paramètres qui expliqueraient aussi la baisse des niveaux de populations que nous n'avions pas pris en compte dans les analyses.

Par exemple, pour le niveau zéro de fumure (SF), le facteur temps (le développement des cotonniers au fur et à mesure dans le temps) a permis d'expliquer à $96 \%$ la première phase de l'accroissement des populations et $82 \%$, celui de la deuxième phase. Par contre ce même facteur n'a permis d'expliquer qu'à 89 et 77 $\%$ et, dans le même ordre, la baisse du niveau de populations au cours des deux phases de diminution de la population. La même tendance a été observée avec les autres traitements mais avec de légères différences quant au niveau d'explication de la variation des niveaux de populations par le facteur temps par rapport aux facteurs qui ne sont pas pris en compte dans l'analyse (Figs. 4 - 7).

Les équations de régression des différentes phases d'accroissement des populations ont montré que l'augmentation du nombre de pucerons dans le temps a été plus élevée lorsqu'on passe des plants de cotonniers sans fumure minérale aux cotonniers avec la forte dose de fumure. En effet, le modèle a montré qu'à chaque 3 jours, on a 48 pucerons de plus 

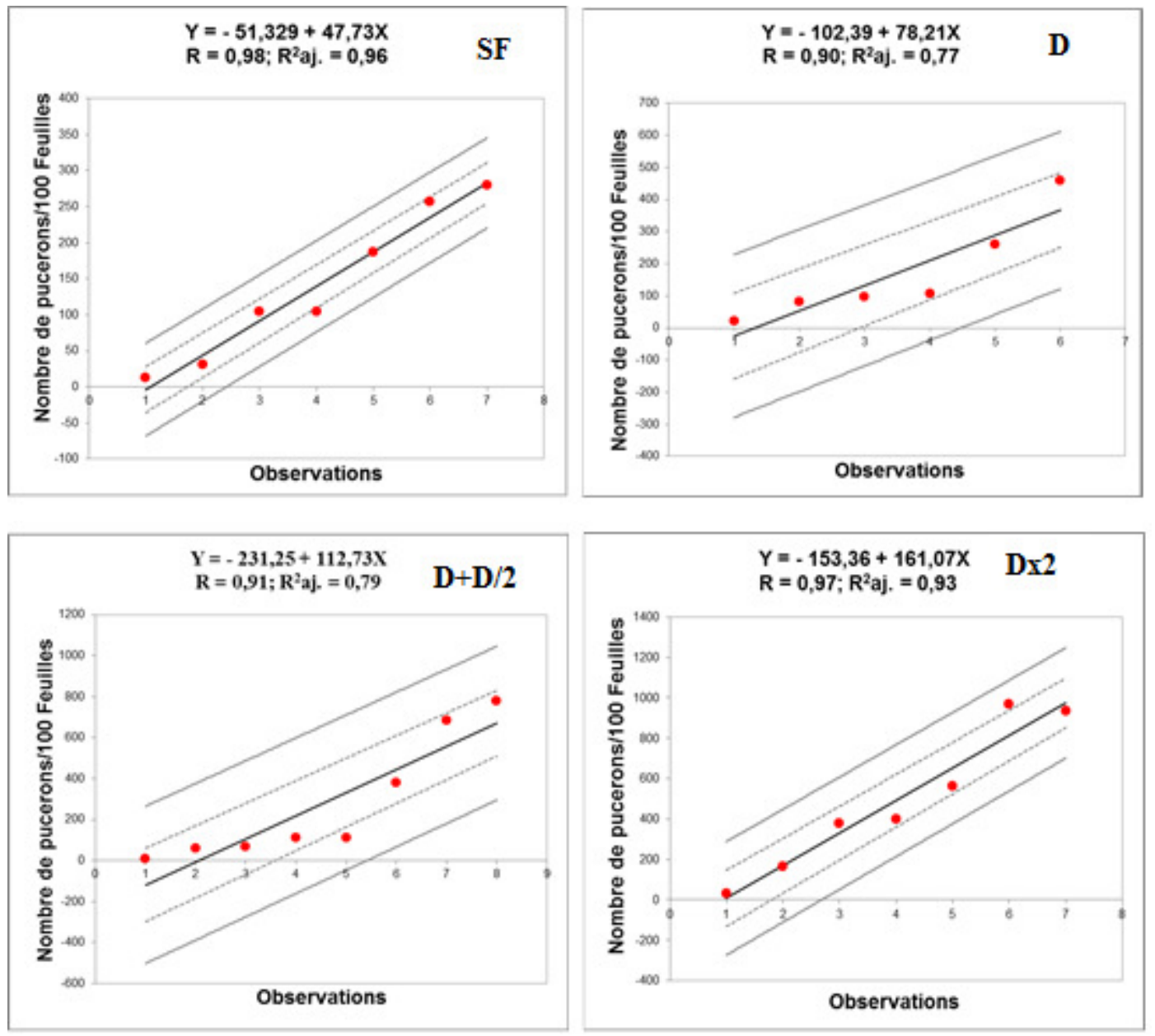

Figure 4. Droites de régression d'accroissement des populations au stade de croissance des cotonniers.

pour le traitement SF, 78 pucerons pour D,

Effets de quelques prédateurs sur la 113 pucerons pour $\mathrm{D}+\mathrm{D} / 2$ et 161 pucerons pour Dx2 (Fig. 4). Les mêmes tendances ont été observées au cours de la deuxième phase d'accroissement de populations (Fig. 6). La première période de baisses de populations a été plus forte pour les traitements qui ont reçu de fortes doses de fumure minérale, $\mathrm{D}+\mathrm{D} / 2$ et Dx2 (Fig. 5). Mais les baisses de niveaux de populations en fin du cycle du cotonnier sont plus élevées en passant des cotonniers sans fumure minérale aux cotonniers avec la forte dose de fumure minérale (Fig. 7).

fluctuation de population d'A. gossypii suivant les doses de fumure minérale. La présence des prédateurs (coccinelles, syrphes et chrysopes) associés à la fluctuation de populations des pucerons a été remarquable. Les courbes de tendance ont montré que les populations de prédateurs ont varié en fonction de celles des pucerons (Fig. 8). L'augmentation de la population de ces derniers a induit celle des prédateurs et inversement. En général, sur tous les traitements, les pics de pullulation des prédateurs correspondent 

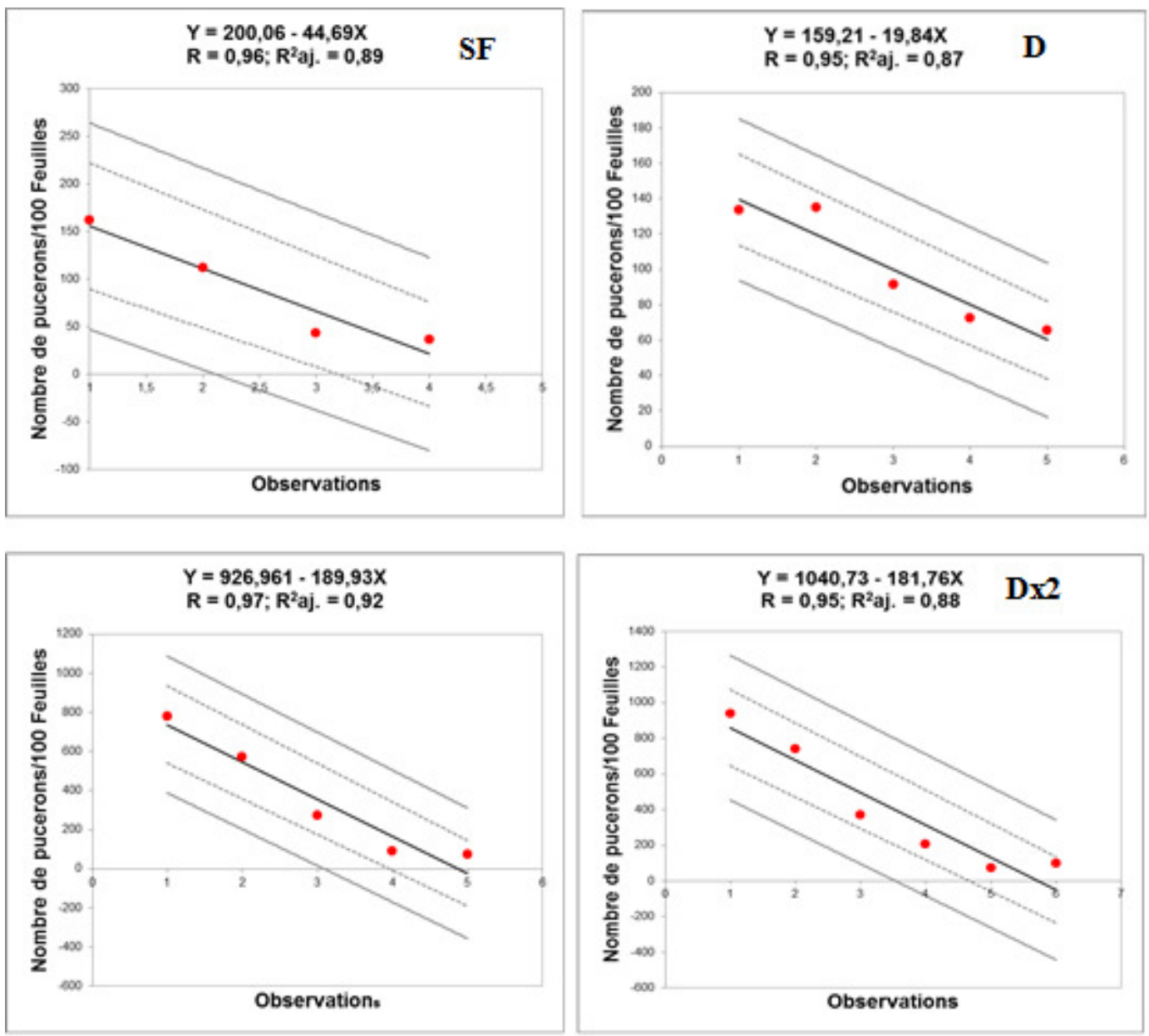

Figure 5. Droites de régression de la baisse du nombre de populations de pucerons au stade croissance et debut de fructification des cotonniers.

aux phases de pullulation de pucerons. Mais leurs effets dépressifs n'ont pas été perceptibles sur la seconde période de pullulation.

\section{DISCUSSION}

L'infestation des plants de cotonniers par $A$. gossypii dans cette étude a varié dans le temps. Elle est caractérisée par deux pics au cours du cycle du cotonnier : (i) le premier pic a été observé à partir du $46^{\text {eme }}$ jas et (ii) le deuxième pic plus important à partir de $86^{\mathrm{eme}}$ jas selon les traitements. Ces résultats confirment ceux de Akantetou et al (2012). Pour les auteurs la population de pucerons augmente à la maturité et à l'ouverture des capsules et fait progressé le pourcentage de coton collant au Togo (ITMF, 2004). Egalement, les populations d' $A$. gossypii de la grande période de pullulation du deuxième pic causent indirectement des dégâts économiquement très considérable (Deguine et Ferron 2004).

Les niveaux de pullulations ont été très dépendants de la dose de fumure minérale appliquée (Fig. 2). Tout en étant économiquement important, ces niveaux des populations de pucerons sur les plants du cotonnier du traitement sans fumure minérale ont été inférieurs, tout le long du cycle de la 

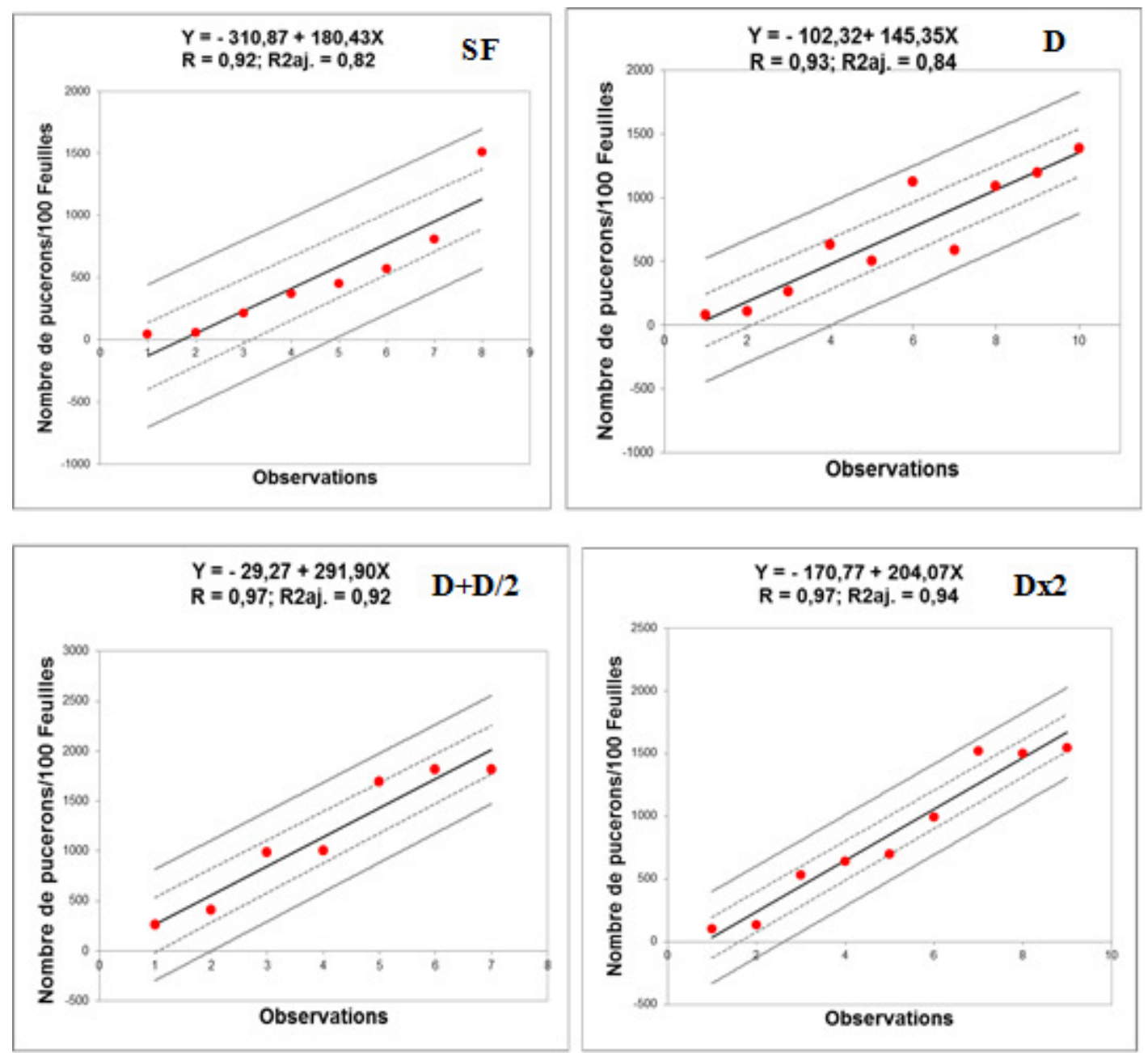

Figure 6. Droites de régression d'accroissement des populations au stade de fructification des cotonniers.

culture cotonnière, à ceux des traitements qui ont bénéficié de l'apport de la fumure minérale.

L'accroissement de la population de puceron observé au cours de la phase de croissance du cotonnier suivant les traitements peut s'expliquer par le caractère intrinsèque du puceron qui lui permet de réagir conformément à la disponibilité ou au manque de ressources alimentaires. Avec un coefficient de multiplication rapide, A. gossypii a été favorisé par la disponibilité de la sève abondante à cette période de croissance des cotonniers au niveau des plants qui ont bénéficié de l'apport de l'engrais minérale. Les différences observées dans l'accroissement des populations suivant les traitements et le temps mis pour atteindre un premier pic dénotent donc de la différence de disponibilité et probablement de qualité nutritive de la sève au niveau des plants de chaque traitement (Deguine et Hau, 2001).

En effet, en absence de fumure minérale, la multiplication des pucerons a été lente et le premier pic n'a été observé qu'au $49^{\text {ème jas }}$ avec un nombre moyen de 280 pucerons par 100 feuilles de cotonnier (Fig. 2 courbe SF). A ce stade de développement des plants, chaque jeune feuille étant très petite et moins 

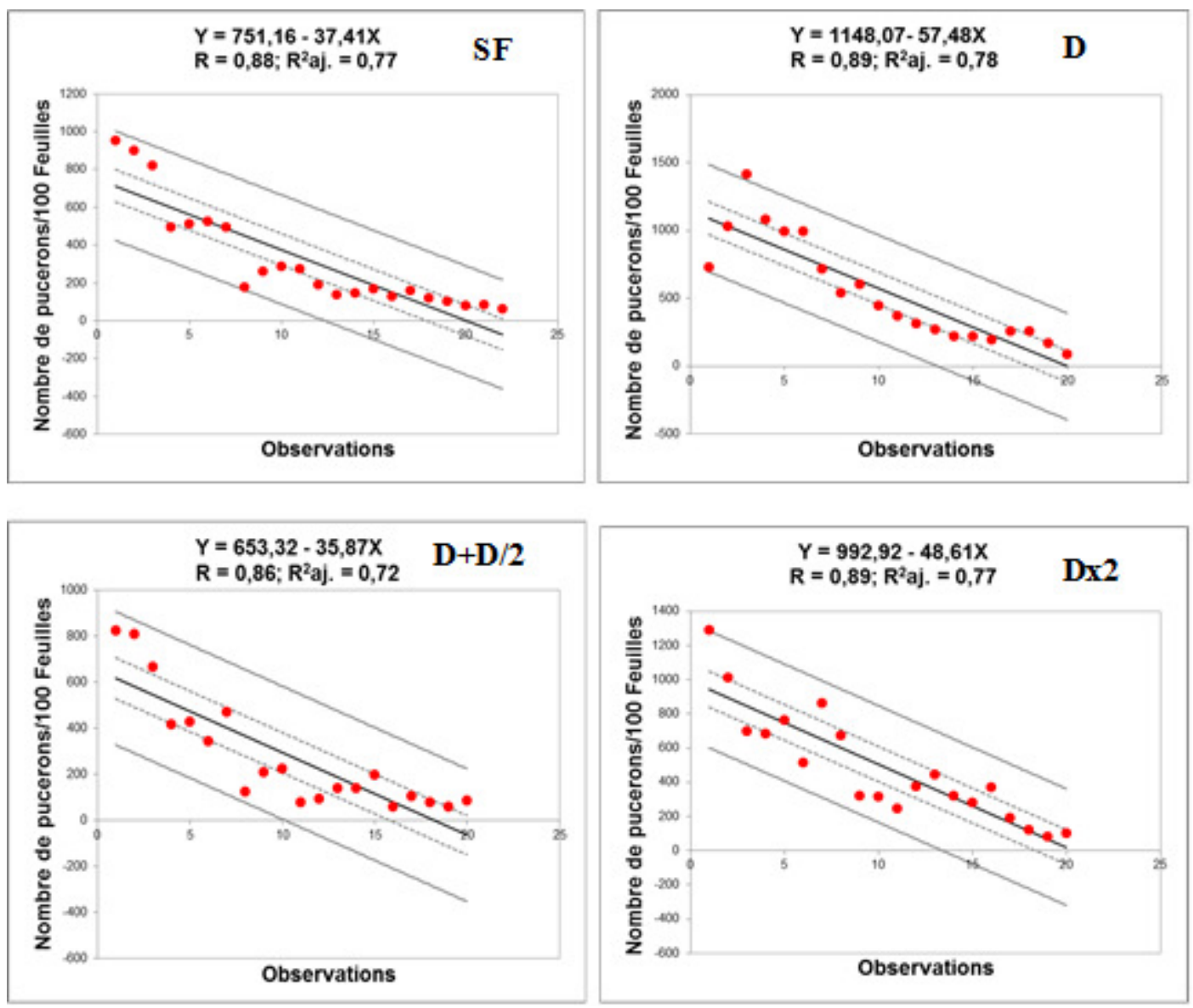

Figure 7. Droites de régressions de la baisse du nombre de populations de pucerons au stade maturité des capsules et fin du cycle des cotonniers.

pourvue en sève était parasitée par 3 pucerons en moyenne. A partir de ce pic, il y a eu diminution du nombre de pucerons à cause éventuellement de l'insuffisance alimentaire ne permettant pas l'augmentation ou le maintien du niveau d'infestation. Dans ces conditions, les pucerons migrent vers d'autres parcelles à la recherche d'autres sources d'alimentation (Deguine, 1995).

Les parcelles ayant reçu la fumure minérale à la dose recommandée aux producteurs de coton (D) ont été rapidement infestées avec un niveau de populations plus élevé que les parcelles avec zéro apport de fumure minérale . Le premier pic a été observé au $46^{\text {ème jas }}$ avec 460 pucerons par 100 feuilles de cotonniers soit 5 pucerons en moyenne par feuille. Par contre, le traitement qui a réçu la dose recommandée plus la moitié $(\mathrm{D}+\mathrm{D} / 2)$ a connu une infestation plus lente que celle des parcelles avec zéro apport de fumure minérale et le premier pic d'infestation est observé au $52^{\text {ème }}$ jas, mais avec une population de 781 pucerons sur 100 feuilles soit 8 pucerons en moyenne par feuille. Sur ces parcelles l'infestation a été lente mais la multiplication des pucerons a été par la suite rapide. Les cotonniers qui ont reçu la double dose de fumure minérale (Dx2) ont connu un premier pic d'infestaion au $46^{\text {ème }}$ jas avec une population de 967 pucerons sur 100 feuilles soit 10 pucerons en moyenne par feuille. 


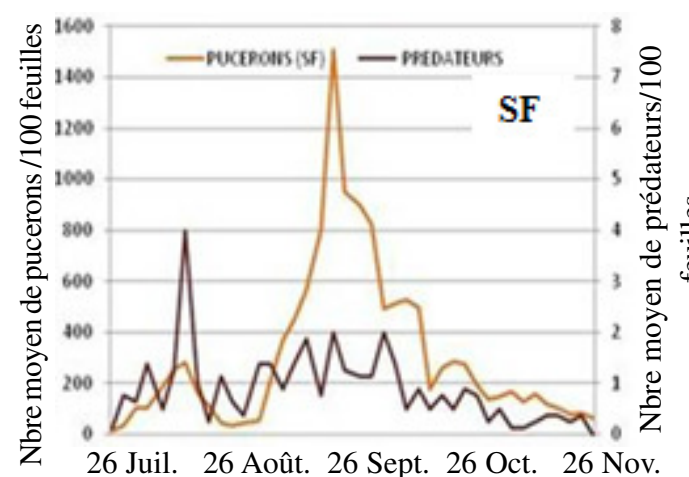

Dates des observations

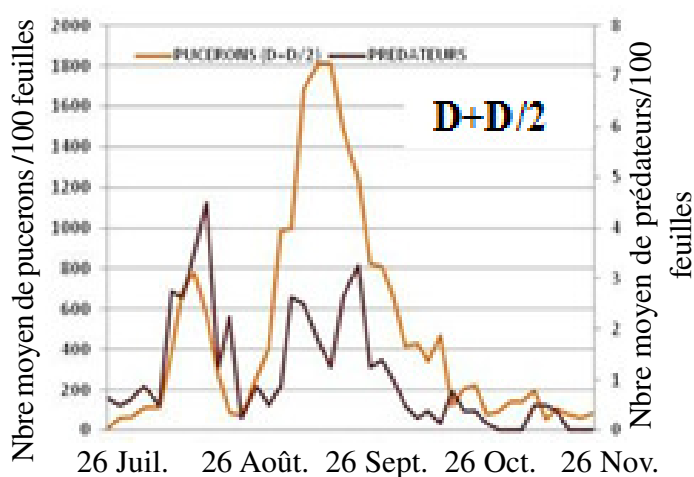

Dates des observations

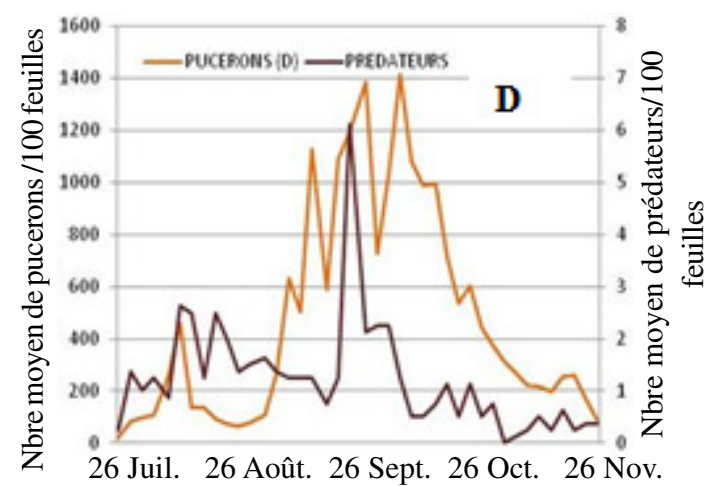

Dates des observations

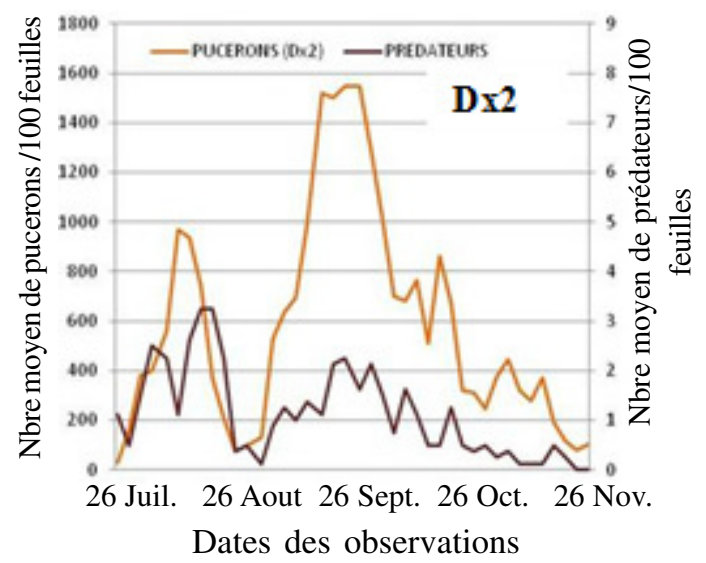

Figure 8. Fluctuation de la population de pucerons et des prédateurs dans les différents traitements.

La différence de temps mis pour atteindre un maximum de populations supportable par la plante suivant le traitement est due à la disponilité et à la qualité de la sève. En effet, l'atteinte rapide du pic ne signifie pas que ces cotonniers sont plus infestés. Le nombre de pucerons observé sur les parcelles ayant reçu la fumure minérale peut être supérieur à celui du pic des parcelles avec zéro fumure minérale. Compte tenu de la disponibilité de la sève au niveau des plants des parcelles ayant reçu la fumure minérale, le nombre de pucerons pic observé au niveau des différentes traitements a été croissant lorqu'on passe du traitement avec zéro fumure minérale (280 pucerons). Ce nombre est multiplié par 1,6 sur les plants des parcelles qui ont reçu la fumure minérale à la dose recommandée, par 2,8 pour la $\mathrm{D}+\mathrm{D} / 2$ et 3,6 pour la double dose (Dx2).
Le rythme de la baisse de populations de pucerons après le premier pic a été également différent au niveau de chaque traitement. La population de pucerons a été de 36 au minimum après le premier pic au niveau du traitement sans fumure minérale. Elle a été de $66 ; 72$ et 74 pucerons respectivement pour les traitements $\mathrm{D}, \mathrm{D}+\mathrm{D} / 2$ et $\mathrm{Dx} 2$ en moyenne sur 100 feuilles de cotonniers (Fig. 2).

La reprise de l'accroissement de la population du puceron, après la baisse qui a suivi le premier pic, a conduit au deuxième pic marquée par un nombre de pucerons supérieur à 1000 pucerons par 100 feuilles pour tous les traitments. Cependant, la diffférence entre les traitements a été observée au niveau du maintien du pic dans le temps. En effet, pour le traitement avec zéro fumure minérale, l'acroissement a repris d'une manière 
exponentionnelle mais pour une courte durée puis la baisse a été rapide jusqu'à un niveau bas avec 64 pucerons observés sur 100 feuilles subterminales au $150^{\text {ème }}$ jas du cotonnier. Les traitements avec apport de la fumure minérale ont conduit à un deuxième pic un peu plus étalé dans le temps suivant la dose appliquée et le nombre de pucerons observé a été plus élevé aux dernières observations, $89 ; 85$ et 101 pucerons respectivement pour la $\mathrm{D}$, la $\mathrm{D}+\mathrm{D} / 2$ et la Dx2 (Fig. 2).

La non différence significative entre les niveaux d'infestation des cotonniers observée au cours du premier mois de la phase de croissance des cotonniers (30 ème jas) peut s'expliquer par le fait que des pucerons ont été observés seulement sur quelques feuilles des plants de cotonniers. Ces pucerons aptères proviennent des premières générations des pucerons ailés qui ont migré pour coloniser la parcelle nouvellement mise en place. Par contre, les différences très significatives entre les niveaux de populations observés sur les plants de cotonniers des différents traitements

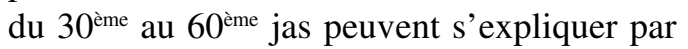
la différence de développement des plants suivant les traitements suite à l'apport de la fumure à différentes doses au $20^{\text {ème }}$ jas pour

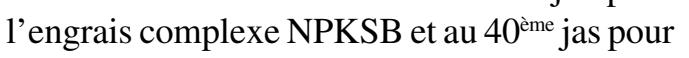
l'Urée. La double dose de fumure minérale a permis une infestation moyenne, nettement supérieure aux infestations observées sur les plants des autres traitements mais statistiquement équivalente à celle observée sur les parcelles de la dose de fumure minérale $\mathrm{D}+\mathrm{D} / 2$. D'une manière générale, le niveau de populations de pucerons a été croissant et en corrélation avec l'augmentation de la dose de fumure minérale apportée.

Au stade floraison et fructification du $60^{\text {ème }}$

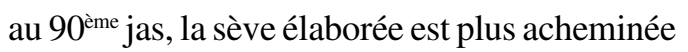
vers les feuilles qui alimentent les organes fructifères au détriment de la croissance de la plante. La quantité de sève élaborée qui arrive au niveau des feuilles dans la partie apicale de la plante n'est pas importante.
Le puceron a réagi donc au phénomène en réduisant éventuellement son potentiel de reproduction avec diminution du nombre de pucerons sur les jeunes feuilles terminales sur lesquelles sont réalisées les observations.

A la fin de la période de fructification et à l'ouverture des capsules, les plants du traitement SF ont connu un niveau d'infestation nettement inférieur à ceux des plants des traitements ayant reçu la fumure minérale. Ceci explique l'intérêt de la fumure minérale dans la disponibilité et l'amélioration de la qualité de la sève élaborée au niveau des plants de cotonnier qui favorisent le développement des pucerons sur une période plus longue que lorsque les plants n'ont pas reçu l'application de la fumure.

Les équations de régression des différentes phases d'accroissement des populations ont également montré que l'augmentation du nombre de pucerons dans le temps a été plus élevée lorsqu'on passe des cotonniers sans fumure minérale aux cotonniers ayant reçu la plus forte dose. En effet, le modèle a montré qu'à chaque 3 jours, on a respectivement 48 pucerons de plus pour le traitement SF, 78 pour D, 113 pour D+D/2 et 161 pour Dx2 (Fig. 4). Les mêmes tendances sont observées au cours de la deuxième phase d'accroissement de populations. La première période de baisses de populations a été plus forte pour les objets qui ont reçu de fortes doses de fumure minérale, D+D/2 et Dx2 (Fig. 5). Mais les baisses de niveaux de populations en fin du cycle du cotonnier sont plus élevées en passant des cotonniers sans fumure minérale aux cotonniers ayant reçu la plus forte dose de fumure minérale (Fig. 7).

Ces résultats obtenus en conditions naturelles (au champ) confirment ceux obtenus en laboratoire par certains auteurs sur l'effet favorable des fortes doses de fumure sur la pullulation d'A. gossypii sur le cotonnier (Celeni, 2001 ; Deguine et Hau , 2001). En effet, Deguine et Hau (2001) ont rapporté que les taux de certains composants comme les 
acides aminés et les sucres peuvent définir en partie le potentiel de développement d'A. gossypii.

A chaque pic, il y a eu réduction du potentiel de reproduction des pucerons et éventuellement des migrations des adultes aptères des plants saturés vers les plants proches moins infestés.

La naissance des pucerons ailés facilite leur migration plus loin pour coloniser de nouvelles zones alimentaires. En effet, cette espèce peut produire des individus aptères et ailés dans la même population. La dispersion par la marche se fait par les aptères qui migrent à l'échelle locale intra-plant (entre les feuilles d'un même plant) et inter-plants (entre proches voisins) quand la densité locale augmente. La propagation de cotonnier à cotonnier est assurée par les générations successives d'aptères (Deguine, 1995).

Quand les conditions deviennent défavorables, et plus généralement quand la densité locale devient trop forte, des ailés sont produits pour assurer une dispersion plus large (Lombaert et al., 2006). Dans une étude sur la dispersion d'A. gossypii sur plants de melon, Lombaert et al. (2006) ont confirmé que les processus de dispersion seraient densitédépendants : quand la densité locale dépasse un certain seuil (d'environ 6000 pucerons par plant), une proportion de la population disperse par la marche, et quand un seuil supérieur est atteint (environ 10000 pucerons par plant), des ailés sont produits.

Il est également possible que la baisse des populations intervenue après les pics soit accélérée par l'action des facteurs abiotiques (pluviosité, température, humidité relative, etc.) et biotiques (ennemis naturels). Car, les insectes antagonistes d'A. gossypii arrivent deux à trois semaines après l'infestation de la culture par les pucerons (Celeni, 2001).

Prédateurs. Bien que, sur tous les traitements, les pics de pullulation des prédateurs correspondent aux phases de pullulation de pucerons leurs effets dépressifs n'ont pas été perceptibles au cours de la grande période de pullulation des pucerons (Fig. 8). Après l'infestation des plants de cotonniers par les pucerons, les coccinelles et les syrphes ont été les premiers à être présents dans les parcelles puis les chrysopes ont suivi et ont eu une action de régulation précoce et efficace sur les premières pullulations. L'action précoce des coccinelles et des prédateurs des pucerons a été aussi démontrée dans d'autre pays (Ronzon, 2006, Belhadi et al., 2011). En effet, ces auteurs ont montré que les coccinelles étaient précoces et ont une action de régulation efficace sur les premières pullulations de pucerons mais deviennent moins actives en cas de forte pullulation des pucerons. L'effet non dépressif des prédateurs sur la population des pucerons dans la grande période de pullulation peut trouver son explication dans les résultats rapportés par Ferguson (2005). Pour l'auteur, les prédateurs de pucerons font généralement preuve de moins de discrimination que les parasites et se nourrissent d'une large gamme d'autres espèces. Ils sont plus efficaces lorsque la population de pucerons est moins importante et moins efficaces en cas de forte pullulation. Aussi, lorsqu' on compare les temps de développement des pucerons et leurs ennemis naturels, à l'instar des coccinelles, on constate que les premiers se développent plus vite que les seconds. Cette différence de vitesse de développement qui confère aux pucerons leur caractère de proie éphémère pour les coccinelles ou limitée dans le temps. Egalement, le calcul du rapport du temps de génération des prédateurs sur celui des pucerons, «Generation Time Ratio », est supérieur à 1 . Or, les rapports supérieurs à 1 correspondent à des relations trophiques défavorables à la mise en place d'une régulation « Top-down » (Ndzana Abanda, 2012). En plus, les prédateurs sont des ennemis naturels non spécifiques uniquement aux pucerons mais disposent d'une gamme de proies au choix. Leur présence élevée sur cotonnier n'est pas corrélée à leur impact sur les pucerons. D'où leur limite dans la régulation 
des populations de pucerons dont la vitesse de multiplication est aussi plus rapide.

Pour réguler les populations d'A. gossypii en culture cotonnière, il faudrait envisager d'autres possibilités de lutte biologique en plus de celles des ennemis naturels et éventuellement l'action des facteurs climatiques (Deguine et Vaissayre, 2000). Car, avec les phénomènes de changement climatique affectant la régularité des pluies, l'action du champignon phytopathogène Neozygites fresenii (Nowakowski) qui régule les pucerons en périodes d'abondantes pluies (Deguine, 1995) n'est plus assurée. L'approche par le «Bottonup » qui privilégie l'augmentation de la biodiversité végétale en champ pour réguler la dynamique de populations du puceron (Ndzana Abanda, 2012) et l'utilisation des biopesticides dans le cadre d'une lutte intégrée seraient bien indiquées.

\section{CONCLUSION}

L'étude comparative des courbes de tendance, des histogrammes et les droites de régression des variations mensuelles des populations de pucerons, suivant les différentes doses de fumure minérale, a montré que les niveaux d'infestation des plants sans fumure minérale (SF) ont été inférieurs à ceux des traitements ayant reçu la fumure minérale. Les plants de cotonniers des parcelles qui ont reçu la plus forte dose de fumure minérale (Dx2) ont connu un deuxième pic qui s'est étalé sur une période de près d'un mois. L'importance des infestations et les niveaux de variation de populations au cours du cycle de la culture cotonnière ont souvent corrélés avec les doses de fumure minérale appliquées. Dans l'optique de la gestion agronomique d'A. gossypii, l'utilisation optimale des éléments fertilisant est l'idéale pour éviter ses fortes infestations de culture à cause de l'abondance de la sève élaborée et disponible. La présence et le développement des prédateurs (coccinelles, syrphes et chrysopes) ont évolué en fonction du niveau d'infestation des plants par le puceron sans avoir un effet régulateur remarquable sur ces derniers. Ces résultats montrent qu'avec les variations climatiques entrainant des conditions beaucoup plus favorables au développement d'A gossypii, en plus de son potentiel intrinsèque de multiplication rapide, les programmes classiques mis au point pour son contrôle en culture cotonnière ne seront plus indiqués à court ou à moyen terme où de fortes infestations en début du cycle ont nécessité des traitements avec des produits aphicides avant la période prévue dans le programme. La mise au point d'une stratégie de gestion intégrée de ce ravageur devient nécessaire. L'utilisation optimale aux dates recommandées de la fumure minérale peut permettre de réduire le niveau des infestations.

\section{REMERCIEMENT}

Les auteurs remercient les responsables de la Station d'Expérimentation Agronomique de l'Université de Lomé (SEAL) Togo pour la mise à disposition du site qui a permis la réalisation des différents travaux dans le cadre de cette étude. Les remerciements sont adressés également à Monsieur Sekaya Rigobert A. pour son dévouement dans l'entretien de la parcelle expérimentale.

\section{REFERENCES}

Akantetou, K.P., Koba, K., Poutouli, W.P., Ayeva, B., Bonfoh, B. et Sanda, K. 2012. Niveau d'infestation des populations d'Aphis gossypii Glover (Homoptera : Aphididae) en culture cotonnière au Togo. Cameroon Journal of Biological and Biochemical Sciences 20:30-41.

Akantetou, K.P. 2014. Etude de la dynamique de population d'Aphis gossypii Glover, 1877, ravageur du cotonnier (Homoptera: Aphididae) et potentiel aphicide des huiles essentielles d'Ocimum canum Sims et d'Ocimum basilicum L. (Lamiaceae). Thèse de Doctorat, Université de Lomé, Togo, $195 \mathrm{pp}$. 
Belhadi, A., Djoudi, M., Berredjouh, D. et Baazi, K. 2011. Des insectes auxiliaires autochtones à protéger et à valoriser pour une agriculture saine dans des régions à agro-écosystèmes vulnérables. Centre de Recherche Scientifique et Technique sur les Régions Arides Omar Elbarnaoui (CRTSTRA). 6pp.

Bergé, J.B. et Ricroch, A.E. 2010. Emergence of minor pests becoming major pests in GE cotton in China. What are the reasons? What are the alternative practices to this change of status? GM Crops 1:214-219.

Célini, L. 2001. Le puceron du cotonnier Aphis gossypii (Glover) et son parasite Aphelinus gossypii Timberlake en République centrafricaine. $4 \mathrm{p}$.

Decoin, M., Lerin, J., Chabert, A., Van Waetermeulen, X., Lapierre, H., Dederyver, D. et Caubel, G. 1999. Connaître les dynamiques de population de ravageurs : Modéliser, prévoir, pour mieux raisonner la protection en grandes cultures : Ravageurs $=$ Awareness of the dynamics of pest populations. Phytoma 522:27-33.

Deguine, J.P. 1995. Bioécologie et épidémiologie du puceron Aphis gossypii Glover, 1877 (Hemiptera, Aphididae) sur cotonnier en Afrique centrale. Vers une évolution de la protection phytosanitaire. Thèse de Doctorat de l' ENSA, Montpellier (Résumé de l'auteur).

Deguine, J.P. et Vaissayre, M. 2000. Proposition pour une gestion durable des populations de puceron, d'aleurodes chez les petits producteurs de coton africain. Acte de la Réunion Phytosanitaire Coraf Réseau Coton. 22-25 Février 2000 Lomé (Togo) pp. 209-218.

Deguine, J.P. et Hau, B. 2001. The influence of the plant on Aphis gossypii. Some results of research conducted in Cameroon. Ciradca, Cotton Program, TA 72/09, 34398 Montpellier Cedex 5, France. Proceedings of the seminar, July 4-7, 2001, Lille, France.

Deguine, J.P. and Ferron, P. 2004. Protection des cultures et développement durable bilan et perspectives. Courrier de l'environnement de l'INRA $\mathrm{n}^{\circ} 52$, septembre 2004. CIRAD, 34398 Montpellier cedex 5. pp. 57-65.

Ferguson, G. 2005. Facteur à considérer dans l'usage d'agents de lutte biologique contre les Pucerons. http://www.search.gov.on. ca: $8002 /$ cumpass ? view-template $=$ simple 1 Ontario Ministère de l'Agriculture, de l'Alimentation et des eaux

Frydrych, R. et Hequet, E. 1997. Les cotons collants, Fascicule de formation, CIRAD, 98pp.

Hofs, J.L., Goze, E., Cene, B., Kioye, S.et Adakal, H. 2013. Assessing the indirect impact of Cry1 Ac and Cry2Ab expressing cotton (Gossypium hirsutum L.) on hemipteran pest populations in Burkina Faso (West Africa). GMOs in integrated plant production. IOBCWPRS, Bulletin 97: 4954.

ITMF. 2004. Cotton contamination surveys 1999 - $2001-2003,21$ p. Site web: http:// www.itmf.org.

ITMF. 2011. Cotton contamination surveys 1991 -2011, 21 p. :http://www.itmf.org.

Lombaert, E., Boll, R. et Lapchin, L. 2006. Dispersal strategies of phytophagous insects at a local scale: adaptative potential of aphids in an agricultural environment. BMC Evolutionary Biology pp. 6- 75. 1 à 13. è'10.1186/1471-2148-6-75é'

MAEP, 2013. Document d'orientation stratégique de la filière coton au Togo. 60pp.

Miranda, J.E., Rodrigues, S.M.M., Almeida, R.P., Sylva, C.A.D., Togola, M., Hema, S.A.O., Somé, N.H., Boni, G., Adegnika, M.O., Doyam, A.N. et Diambo, B. 2013. Reconnaissances de ravageurs et ennemies naturels pour les pays C-4. Embrapa, Brésil. 70pp.

Ndzana Abanda, X.F.R. 2012 Régulation des bio-agresseurs dans les cultures associées de blé dur et de pois: impact de la diversité végétale sur la démographie des pucerons du pois. Thèse de Doctorat de l'Université de Toulouse. 141pp. 
NSCT. 2013. Quelques éléments de synthèse sur la campagne 2012/2013. Communication de la Direction de Soutien à la Production (DSP) à la réunion de concertation pour la préparation et le lancement de la campagne de production cotonnière 2013/2014, Kara, 23 et 24 mai 2013.
Ronzon, B. 2006. Biodiversité et lutte biologique. Comprendre quelques fonctionnements écologiques dans une parcelle cultivée, pour prévenir contre le puceron de la salade Extrait d'un mémoire de fin d'étude sur les bandes fleuries, qui sont utilisées comme réservoir d'insectes auxiliaires, ENITA de Clermont Ferrand, 25pp. 